NASA/TM-2001-211155

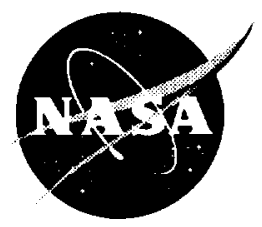

\title{
Verifying Data Integrity of Electronically Scanned Pressure Systems at the NASA Glenn Research Center
}

Joseph W. Panek

Glenn Research Center, Cleveland, Ohio

Prepared for the

2001 19th International Congress on Instrumentation in

Aerospace Simulation Facilities (ICIASF 2001)

cosponsored by the IEEE AES, NASA Glenn, and OAI

Cleveland, Ohio, August 27-30, 2001

National Aeronautics and

Space Administration

Glenn Research Center 


\section{Acknowledgments}

Even though he has been retired for almost 10 years, I would like to acknowledge Steve Gonczy for his development of the Continuity/Leak Checking and Check Pressure Processes. These were developed in the 1980's for the first generation ESP System. It is a tribute to Steve's attention to detail and forethought that these techniques are still current and effective. I would like to thank the following individuals for their expert and timely review of this paper, Robert Freedman, Alan Linne, and Timothy McCartney.

This report contains preliminary findings, subject to revision as analysis proceeds.

Trade names or manufacturers' names are used in this report for identification only. This usage does not constitute an official endorsement, either expressed or implied, by the National

Aeronautics and Space Administration.

Available from

NASA Center for Aerospace Information

National Technical Information Service

7121 Standard Drive

5285 Port Royal Road

Hanover, MD 21076

Springfield, VA 22100 


\title{
VERIFYING DATA INTEGRITY OF \\ ELECTRONICALLY SCANNED PRESSURE SYSTEMS AT THE NASA GLENN RESEARCH CENTER
}

\author{
Joseph W. Panek \\ National Aeronautics and Space Administration \\ Glenn Research Center \\ Cleveland. Ohio 44135
}

\begin{abstract}
The proper operation of the Electronically Scanned Pressure (ESP) Systems at the NASA Glenn Research Center is critical to accomplish the following goals: acquisition of highly accurate pressure data for the development of future aerospace and commercial aviation systems and continuous confirmation of data quality to avoid costly, unplanned, repeat wind tunnel or turbine testing. Standard automated setup and checkout routines are necessary to accomplish these goals. Data verification and integrity checks occur at three distinct stages, pre-test pressure tubing and system checkouts, daily system validation and in-test confirmation of critical system parameters. This paper will give an overview of the existing hardware, software and methods used to validate data integrity.
\end{abstract}

\section{BACKGROUND}

Electronically Scanned Pressure systems have been in use at NASA Glenn since the late 1970's to measure large numbers of pressures with high accuracies. The classic and the newer Digital Thermal Compensation (DTC) pressure scanners are currently being utilized. Both styles of scanner have an array of silicon pressure transducers that are multiplexed, amplified, scanned by an analog to digital converter and converted to engineering units. An integral, pneumatic calibration valve allows the scanners to be insitu calibrated by a secondary pressure standard. Periodic calibrations maintain system accuracy. For the classic scanner, three to five precision pressures are sequentially applied to all of the transducers and new coefficients are calculated. The general equation for a standard five point ESP calibration is

$$
\mathrm{P}=\mathrm{C} 0+\mathrm{Cl} \mathrm{V}+\mathrm{C} 2 \mathrm{~V}^{2}+\mathrm{C} 3 \mathrm{~V}^{3}+\mathrm{C} 4 \mathrm{~V}^{4}
$$

where

$V$ is the transducer output voltage

$P$ is the transducer pressure and

$\mathrm{CO}, \mathrm{Cl}, \mathrm{C} 2, \mathrm{C} 3, \mathrm{C} 4$ are the calibration coefficients.
The Digital Thermal Compensation (DTC) scanners have been successfully used in a recent wind tunnel test. These scanners employ technology that allows the transducer temperatures to be measured and used in conjunction with coefficients that are stored in the scanner EEPROM to compensate for the transducers ${ }^{\circ}$ temperature changes. This temperature compensation routine extends the calibration interval of the scanners from less than one hour to as much as four hours. These scanners require only a two-pressure calibration to correct zero and span errors. The general equation for this two point DTC ESP calibration is

$$
P=S\left(P_{C}+Z\right)
$$

where

$S$ is the span adjustment

$\mathrm{Z}$ is the zero adjustment

$P$ is the transducer pressure and

$P_{C}$ is the computed pressure from the internal coefficients.

The checkout and verification of both types of scanners will be addressed.

\section{HARDWARE}

The Electronically Scanned Pressure Systems at the NASA Glenn Research Center use the Pressure Systems Incorporated (PSI) System 8400. ${ }^{2}$ These systems employ up to 1024 pressure transducers in ranges from 0.361 Psid ( $2.5 \mathrm{kPa}$ ) to 500 Psid ( 3500 $\mathrm{kPa}$ ). Periodic insitu calibrations maintain the system accuracy to $\pm 0.05 \%$ of transducer range. Four styles of pressure scanners are currently employed to acquire data; 16 and 32-port rack-mount, 32-port miniature and 32-port miniature digital thermal compensated. The system architecture allows the use of all four concurrently. Miniature scanners are generally located in or near the model or test article. The System Processor (SP), scanner interface, Pressure Calibrate Units (PCU's) and rack-mount scanners are located near the test article in either a temperature-controlled 


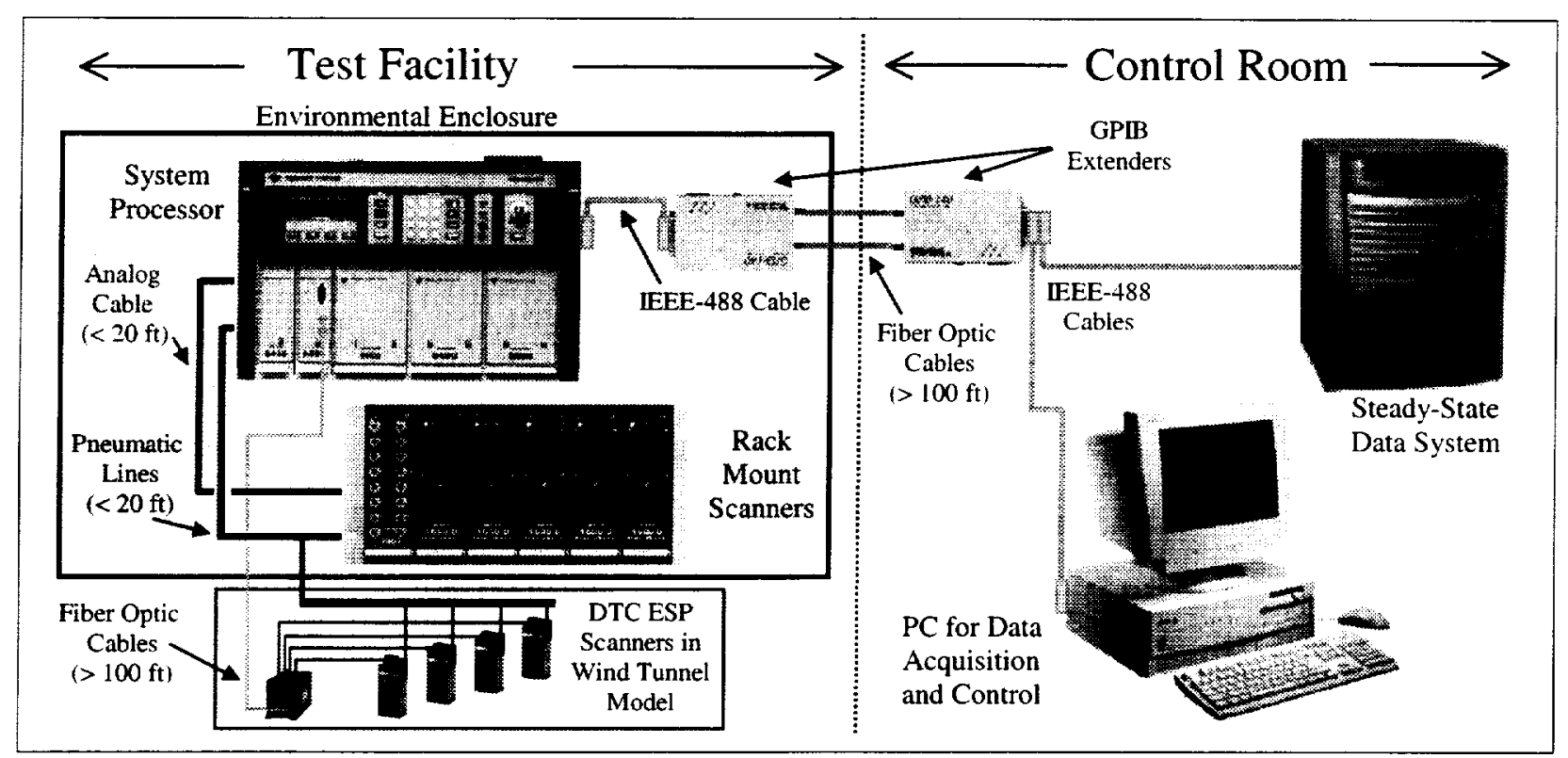

Figure 1. NASA Glenn Research Center Pressure Acquisition System Configuration

environmental enclosure or an air-conditioned room. Keeping the classic pressure scanners at a constant temperature increases the stability of the transducers and decreases the number of calibrations that are required. ${ }^{4}$ The system is controlled via a fiber-optically extended IEEE-488 communication link to the Pentium ${ }^{\mathrm{TM}} \mathrm{PC}$ and Alpha-based steady-state data system computer. These are located in or near the control room. A typical System 8400 wind tunnel installation is shown above in Figure 1.

\section{SOFTWARE}

Standard ESP System software was developed at the NASA Glenn Research Center and is used in over 24 facilities. This software operates on a $133 \mathrm{Mhz}$ (or higher) Pentium ${ }^{\text {TM }}$ PC running the Windows ${ }^{\text {TM }} 95$ or 98 operating system with a commercially available IEEE488 card and driver.

This ESP software, PASCaL (Pressure Acquisition, Setup, Checkout and Logging), consists of three distinct programs; Setup, Checkout and Acquire." The separate programs allow for the optimization of the three primary functions. They share over thirty pages of text and graphical help. On-line log messages document critical system parameters and are written to files for later viewing

PASCaL Setup is used to generate a parameter file that defines the system hardware and data acquisition modes for the other two programs. The program employs algorithms that check for illegal configurations that, if implemented, could damage the equipment. Both graphical and text documentation are generated.

PASCaL Checkout is the primary tool for daily system verification and trouble-shooting. System calibration. pressure scanning, tubing continuity/leak checking and diagnostic routines are automated. This provides a tool for verifying the integrity of the pressure system from the test article pressure tap to the steady-state data system. An automated system checkout routine performs up to six go-no-go user-selectable tests; verification of system temperatures, calibration pressures, transducer coefficients, transducer integrity, tubing continuity and pneumatic calibration integrity. A generated report can be printed or sent to a file. PASCaL Acquire controls scanner calibration, performs post-calibration tests to confirm proper operation and acquires data. This pressure data is automatically corrected for reference pressure changes and passed to the steady-state data system for viewing and use in performance calculations. If the vertical distance from the test article to the pressure scanners is greater than ten feet, the elevation-induced pressures errors can be easily corrected in the steady state data system. ${ }^{3}$

\section{PRE-TEST VERIFICATION AND SYSTEM CHECKOUTS}

\section{Tubing Verification}

Pressure data fidelity is dependent not only upon transducer accuracy but the tubing system that connects 
the transducer to the test article static or total pressure tap. The tubing is verified at the farthest accessible point from the pressure transducer to the transducer itself. Continuity Checks determine if the pressure taps are connected to the proper data channel. Leak Checks measure the tubing leak rate and compare it to predetermined criteria to determine acceptability. The leak-check pressure, $P_{\text {leakCheck, }}$ is applied to a test article static or total pressure tap. The pressure source is manually valved-off to seal the pressure back to the transducer and the trapped pressure is allowed to settle. A continuity check (identification of the port with the highest or lowest pressure) is performed and the system measures the trapped pressure, $P_{0}$. If the pressure is not within the specified tolerance, leak checking is stopped and an error message is generated. After ten seconds, the pressure is again measured and the actual leak rate in Psi/Min is computed.

$$
\text { Leak Rate } \text { Actual }=\left[\mathrm{P}_{\text {tube }}(10)-\mathrm{P}_{0}\right] \cdot 6 \quad \mathrm{Psi} / \mathrm{Min}
$$

where $P_{\text {tube }}(10)$ is the port pressure (pressure trapped in the tube) 10 seconds after the initial pressure reading, $P_{0}$. If the actual leak rate is higher than the maximum acceptable rate, the operator is alerted and the port is flagged in the leak check documentation.

The maximum acceptable leak rate is derived from the conservation of mass. ${ }^{8}$

Vol. $\frac{d p(t)}{d t}+\frac{p(t)-\Delta P u(t)}{1 / L R_{\text {ref }}}=0$

where

$$
\begin{array}{rll}
\mathrm{p}(\mathrm{t}) & =\text { tube pressure at time, } \mathrm{t} & \mathrm{psi} \\
\mathrm{LR}_{\text {ref }} & =\text { Reference Leak Rate } & \mathrm{in}^{3} / \mathrm{sec} . \\
\Delta \mathrm{Pu}(\mathrm{t}) & =\left(\mathrm{P}_{\text {Aum }}-\mathrm{P}_{\text {LeakCheck }}\right) \cdot \text { Unit Step } \mathrm{psi} \\
\mathrm{Vol} & =\text { tube volume }=\left(\pi \cdot \mathrm{D}^{2} \cdot \zeta / 4 \mathrm{in}^{3}\right. \\
\mathrm{P}_{\text {Aum }} & =\text { atmospheric pressure } & \mathrm{psi} \\
\mathrm{D} & =\text { tube diameter } & \text { in } \\
l & =\text { tube length } & \text { in }
\end{array}
$$

The reference Leak Rate, $\mathrm{LR}_{\text {ref, }}$ was chosen to be twice the manufacturer's specification for transducer leak rate. In this case, $\mathrm{LR}_{\text {ref }}=0.000244 \mathrm{in}^{3} / \mathrm{sec}$. To simplify, $\tau=\mathrm{Vol} / \mathrm{LR}_{\mathrm{ref}} \mathrm{sec}^{-1}$, and the solution is

$p(t)=\left[P_{A c m}-P_{\text {LeakCheck }}\right]\left[1-e^{-t / \tau}\right]+P_{\text {LeakCheck }}$ Psi. $^{10}$

Since the leak rate is measured over a ten second interval, the following is evaluated at $t=10 \mathrm{sec}$. to arrive at the maximum acceptable leak rate.

Leak Rate $_{\max }=\left[p(t)-P_{\text {LeakCheck }}\right] \cdot 6 \quad \mathrm{Psi} / \mathrm{min}$
The theoretical pressure error that is introduced in a leaky tube can be calculated. A pressure drop across the tube is induced due to airflow. Since we do not know the exact location of the leak(s), we can assume a worst-case scenario with all the flow occurring through the entire tube. The theoretical measurement error due to the leak can be found using Poiseuille's Law, ${ }^{9}$

$$
\mathrm{P}_{\mathrm{error}}=\frac{128 \mu \ell \mathrm{LR}_{\mathrm{ref}}}{\pi \cdot \mathrm{D}^{4}} \quad \text { Psi }
$$

where $\mu=$ viscosity $=2.7 \cdot 10^{-9} \frac{\mathrm{lb}-\mathrm{sec}}{\mathrm{in}^{2}}$

At the end of leak checking, the tubing is quickly vented to atmosphere. Pressure data is stored during venting to characterize the tubing pressure step response. The data is fit to an exponential function to determine the pressure time constant of the tubing. This data is then used to identify pinched or blocked tubing. The following exponential function is used to determine the pressures for the calculated time constant.

$P_{\text {calc }}(t)=\left[P_{\text {Atm }}-P_{\text {Inital }}\right]\left[1-e^{-/ / \tau}\right]+P_{\text {Initial }} \quad P_{s i}$

where

$P_{\text {calc }}(t)=$ calculated tube pressure at time, $t$ psia

$\mathrm{P}_{\mathrm{Atm}}=$ atmospheric pressure psia

$\mathrm{P}_{\text {Initial }}=$ Initial stored data pressure psia

$\tau \quad=$ computed tube time constant sec. $^{-1}$.

Typical time constant data is shown below in Figure 2. An iterative process is used to compute the pressure time constant. A series of time constants are used to calculate the sum of the difference between the actual and calculated data. When the sign of the sum-of-errors

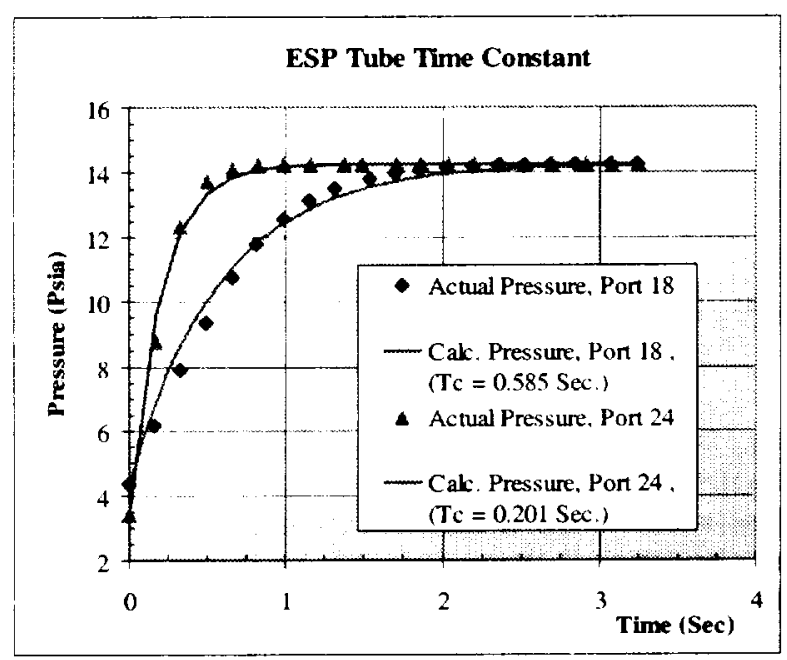

Figure 2. Tubing Time Constant Data 
changes, the final time constant is interpolated between the last two time constants. In Figure 2, it can be seen that the time constant of scanner port 18 is about three times that of port 24 . With the same size tubes, this is indicative of a partially pinched or blocked tube. This method can be used to either identify and repair the defective tube or accept the condition and determine the data settling time that is required after a change in test conditions.

\section{System Noise Checks}

Before a new test program begins, the re-configured ESP hardware needs to be checked for noisy data. Two mechanisms are supplied in the PASCaL Checkout program to establish the data's noise levels. The first is a bar graph display that graphically indicates the mean, maximum, minimum and standard deviations of the selected pressures. Lines on the graph show precisely where the aforementioned statistical variables are located. Scanners that have noisy outputs can be identified very quickly. A color-augmented bar graph demonstrating the statistical noise check is shown below in Figure 3. The second method for locating

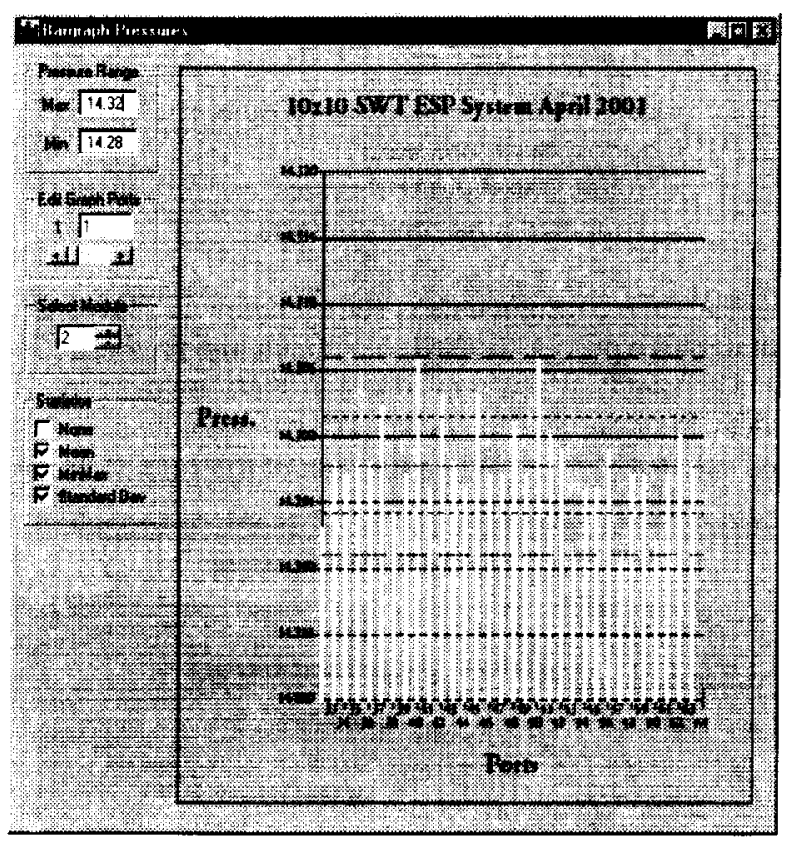

Figure 3. Graphical Statistical Noise Check

noise is an engineering unit window that displays scanner statistics. The mean, maximum, minimum, range and standard deviation of the scanner pressures are calculated. Pressure ports that are known to be bad can be de-selected from the statistic's calculations to properly measure the rest of the scanner. These checks can be used during the daily checkouts.

\section{DAILY SYSTEM VALIDATION}

A number of checks are performed daily before acquiring data. The Checkout and Acquire programs are separate so the acquisition of data can occur with a smaller, more robust, set of code. The Checkout program is run first. At program startup, users are alerted to the calibration status of the Pressure Calibration Units (PCU's). A window warns the user when the PCU calibration expires or is past due. This information is also entered into the system log file.

After calibration, an automated system verification routine checks the PCU(s) temperature(s), calibration pressures' accuracy, transducer coefficients, scanners ' accuracies, pressure ports for plugged tubes and calibration systems leak rates. An example of the Automated System Checkout routine is shown on the following page in Figure 4.

The PCU's temperatures indicate the readiness of the system to acquire data. For most instrumentation, a warm-up period is required to allow for the stabilization of the transducers and associated electronics. If the temperature is close to ambient, the system (including scanners) has probably not had enough warm-up time. The normal operating range is between $80^{\circ} \mathrm{F}\left(26.5^{\circ} \mathrm{C}\right)$ and $105^{\circ} \mathrm{F}\left(40.5^{\circ} \mathrm{C}\right)$. If the temperature is too high ( $>115^{\circ} \mathrm{F},>46^{\circ} \mathrm{C}$ ), a fan may have failed or the system environment may not be suitable. The system log is color coded to denote the severity of problems. In this case, temperatures below $105^{\circ} \mathrm{F}\left(40.5^{\circ} \mathrm{C}\right)$ are black, between $105^{\circ} \mathrm{F}\left(40.5^{\circ} \mathrm{C}\right)$ and $115^{\circ} \mathrm{F}\left(46^{\circ} \mathrm{C}\right)$ are blue and above $115^{\circ} \mathrm{F}\left(46^{\circ} \mathrm{C}\right)$ are red.

The actual calibration pressures that are generated by each PCU are compared to the requested pressures. A calibration pressure tolerance is generally set between $0.05 \%$ and $0.01 \%$ of full-scale pressure. ${ }^{6}$ If the difference between the requested and generated pressure is greater than the tolerance, the calibration is considered to have failed. A failed calibration may still produce what appear to be valid transducer coefficients but signifies that something in the system has changed. A leak may exist in the vacuum or pressure sources.

The transducer coefficients that are computed after the insitu calibration are analyzed to determine if they are within normal limits. The coefficients give the user system "health" information that is very useful for insuring good data.

For the classic (non-DTC) scanners, the criteria on the following page are used for determining "good" coefficients. 


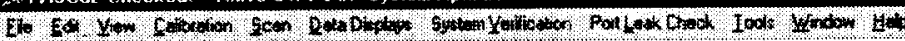

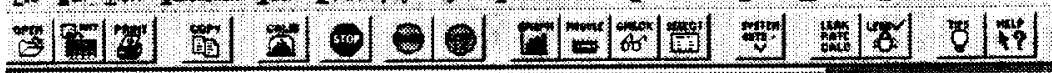

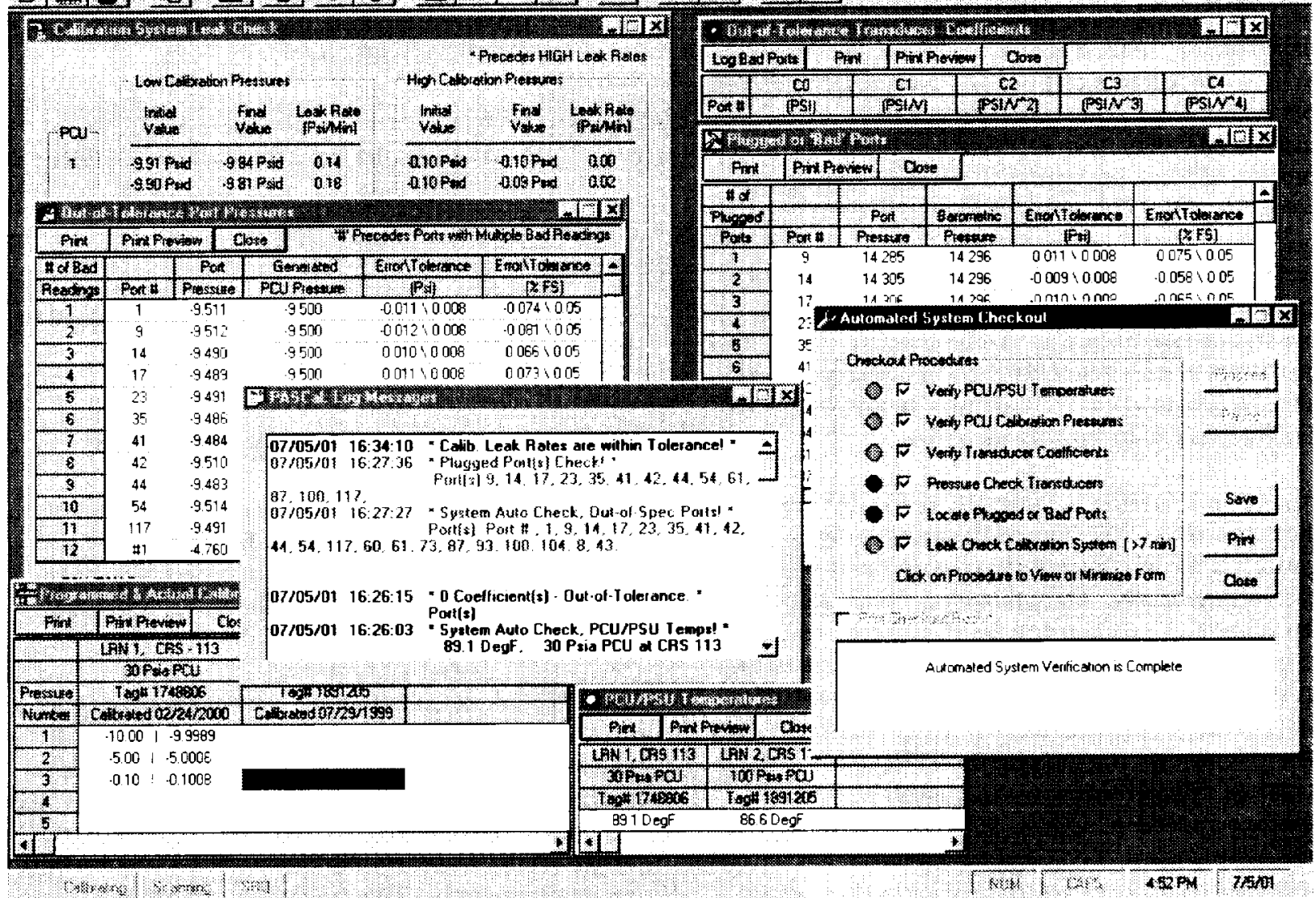

Figure 4. PASCaL Checkout Software - Automated System Checkout

1. The Absolute Value of $\mathrm{C} 0$ should be less than $(\mathrm{Cl} / 2)$ i.e. $\pm 10 \%$ of the scanner's range.

[For an Absolute Calibration, $\mathrm{C} 0$ will be near the reference pressure with a tolerance of $(\mathrm{Cl} / 2)$.]

The CO Coefficient indicates the individual transducer's health. A CO that fluctuates between calibrations is generally an indication of an intermittent transducer. The $\mathrm{Cl}$ Coefficient gives us information about the health of the Scanner Digitizer Unit (SDU), as well as the scanners transducers. If its value is higher or lower than normal, this could signify that the scanner's voltage is not within specifications or that the SDU's A/D converter is not working properly.

2. The Absolute Value of $\mathrm{C} 1 / \mathrm{C} 2$ should be greater than 350 for Scanners $=<100$ Psid.

The Absolute Value of $\mathrm{C} 1 / \mathrm{C} 2$ should be greater than 165 for Scanners $>100$ Psid
The magnitude of the $C 2$ Coefficient indicates the linearity of the calibration. A high C2 generally signifies a leak in or near the calibration pressure line. PCU or scanner. If a single or group of scanners has a C2 that is higher than the others, it is a good assumption that the leak is physically near those scanners. If all scanners have the same magnitude of $\mathrm{C}_{2}$. the leak is probably closer to the PCU.

3. The Absolute Value of $\mathrm{Cl} / \mathrm{C} 3$ should be greater than 1000 for all Scanners Ranges. This tests C3.

4. The Absolute Value of $\mathrm{Cl} / \mathrm{C} 4$ should be greater than $\mathbf{5 0 0 0}$ for all Scanners Ranges. This tests C4.

A high $\mathrm{C} 3$ or $\mathrm{C} 4$ coefficient could indicate leaks that are not linear with pressure. A leak on one side of the reference pressure could be higher than the other. 
For the newer Digital Thermal Compensation (DTC) scanners, a two-point pressure calibration will only adjust the zero and span of the coefficients. Current analytical methods are inadequate to determine if the calibration is valid. Pneumatic leaks in the calibration system can result in coefficients that appear to be valid but produce erroneous pressure readings. A new method had to be devised. This new technique is in the process of being incorporated into the PASCaL software. The DTC scanners require two calibration pressures but will produce valid results with up to five pressures. To minimize the calibration time, three pressures will be utilized. With this new method, calibration non-linearities can be computed, identified and alarmed with generally less than twenty seconds added to the total calibration time. In this way, poor calibrations can be quickly identified.

Another test verifies the integrity of the transducers and the calibration. This transducer pressure check can be performed to determine if the transducers are measuring pressures properly. The calibration valves are placed in the calibration position and the PCU's are commanded to generate a pressure that is $95 \%$ of the value of the calibration pressures. This is to verify near the fullscale values of the scanners but not at the exact values used to calculate the transducer coefficients. Data is acquired from all scanners and compared to the generated pressure that is read by the PCU's secondary pressure standard. Ports that do not match the generated pressure to within $\pm 0.05 \%$ of the scanner range are shown in the Out-of-Tolerance Port Pressure display. This test verifies the integrity of the transducers and the calibration.

Plugged or pinched tubes can be found before testing begins. All ports, except Check Pressures, (see In-Test System Verification for definition) need to be vented to atmosphere. The pressures are then scanned and compared to the lowest range PCU that is reading barometric pressure. If the difference between a port pressure and barometric pressure is greater than $\pm 0.05 \%$ of the scanner range, it is logged.

A calibration subsystem leak-check routine determines its pneumatic integrity. For each PCU. the lowest and highest calibration pressures are re-applied to the system with the scanner calibration valves in the calibration and then run positions. Solenoid valves in the PCU are closed to trap the pressures from the secondary pressure standards to the scanners. The PCU's secondary standard measures the pressures over a 30 second interval and calculates a leak rate in PSI per minute. This leak rate must meet the following manufacturer"s criteria in order to maintain system specifications. ${ }^{11}$
- With the scanner calibration valve(s) in the RUN mode, the leak rate must be less than

$0.5 \%$ of Scanner Range/(Every 32 Ports in the Cal Range) /Minute.

- With the scanner calibration valve(s) in the CAL mode, the leak rate must be less than

$1 \%$ of Scanner Range/(Every 32 Ports in the Cal Range) /Minute.

Calibration ranges that do not meet the criteria need to have the leaks corrected.

\section{IN-TEST SYSTEM VERIFICATION}

Three methods are utilized to insure the detection of problems that may affect quality during data acquisition. The PASCaL Acquire program provides two mechanisms for detecting calibration errors: the third method resides with the steady state data system.

The PASCaL Acquire routines are similar to the ones used in the Checkout program. The generated PCU calibration pressures are checked to insure they are set to the specified values. Out-of-tolerance pressures stop the data acquisition process. It can be assumed that a major change has occurred if the system cannot set the pressures properly. This type of error requires immediate investigation. Transducer coefficients are also verified after every calibration. Out-of-tolerance coefficients are flagged and logged for the user. Bad coefficients do not automatically stop data acquisition. See Figure 5 for examples of out-of-tolerance calibration pressures and transducer coefficients.

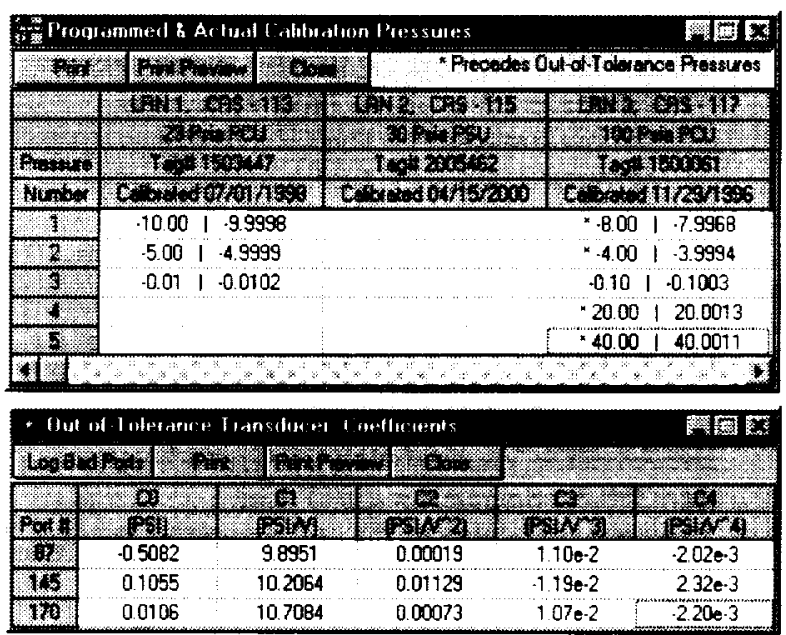

Figure 5. Calibration Pressures and Coefficients 
An applied Check Pressure is the third mechanism for systematic verification of system performance. A known pressure is applied to either the first or last port of every pressure scanner. This known 'Check Pressure" is read with an independent transducer and compared to the scanner ports to check for systematic errors. This comparison is done on the steady state data system. If the Check Pressure is not within $\pm 0.05 \%$ of the scanner's range, an alarm alerts the user that an error has occurred. The system can then be recalibrated to see if the problem can be resolved. If this does not resolve the problem, the PASCaL Checkout program can be used to identify if the source of the error resides in the ESP system or independent check pressure transducer.

\section{CONCLUSIONS}

The techniques used to verify the integrity of the ESP Systems at the NASA Glenn Research Center have resulted in the acquisition of accurate pressure data for its research customers. The continuous confirmation of data has resulted in avoidance of costly unplanned repeat testing. The integrity of the tubing system is quantified from end-to-end. System reliability is enhanced and day-to-day checkout time is reduced by the automated verification of critical system attributes. During data acquisition, the on-line diagnostics indicate current system health and increase the user's confidence in their data.

\section{REFERENCES}

1. ISA Paper \# 2602, "Standard Pressure Acquisition Software at the NASA Glenn Research Center," Joseph W. Panek, Presented at the 46th International Instrumentation Symposium, ISA, May 2000.

2. ISA Paper \# 2603, "Pressure Acquisition System Enhancements at the NASA Glenn Research Center," Joseph W. Panek. Presented at the 46th International Instrumentation Symposium, ISA, May 2000.
3. NASA Technical Memorandum 107240.

"Elevation Correction Factor for Absolute Pressure Measurements," Joseph W. Panek and Mark R. Sorrells, Presented at the 42nd International Instrumentation Symposium. ISA. May 1996.

4. NASA Lewis Research Center, Aeropropulsion Facilities and Experiments Division. Preliminary Information Report \# 064. "Configuring a PSI System 8400 for Maximum Accuracy," Joseph W. Panek, November 1995.

5. ISA Paper \# 0227-7576/97/021-030, "Using Digital Thermal Correction of Electronic Pressure Scanners to Improve Windtunnel Productivity." Harold N. Klaser and Douglas B. Juanarena. Presented at the $43^{\text {rd }}$ International Instrumentation Symposium. ISA. May 1997.

6. "Pressure Systems Incorporated, System 8400 Users Manual,” June 1999. Version 4.0.

7. "How to tell if an ESP Calibration is "Good"." Dan Ridenour. Internal Report, Pressure Systems Incorporated. Hampton, VA, September 1995.

8. Gas Dynamics: Maurice J. Zucrow and Joe D. Hoffman, Copyright 1976, John Wiley and Sons Inc., New York, NY.

9. Modeling Engineering Systems; Jack W. Lewis, Copyright 1994, HighText Publications. Inc., Eagle Rock, VA.

10. Basic Electric Circuit Analysis; David E. Johnson, John L. Hilburn and Johnny R. Johnson, Copyright 1978, Prentice-Hall, Inc., Englewood Cliffs, NJ.

11. Phone conversation with Dan Ridenour, Applications Engineer, Pressure Systems Incorporated, Hampton, VA. 


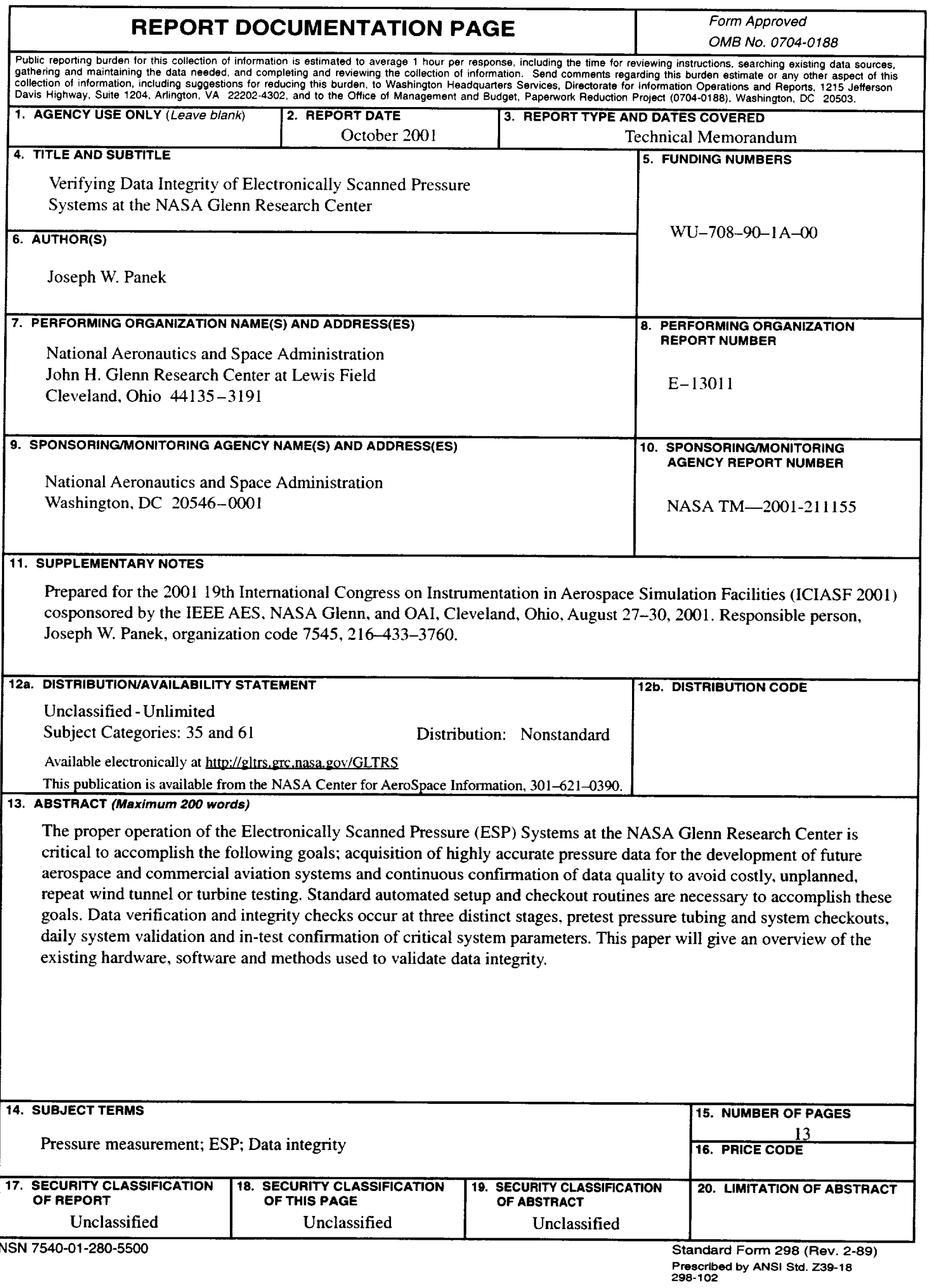

